> Une technologie récente, appelée CRISPR, dérivée du système immunitaire de bactéries, utilise une nucléase Cas9 et un ARN guide complémentaire à une séquence de 20 nucléotides d'un gène pour induire des cassures double brin dans I'ADN. Cela permet de modifier spécifiquement le gène ciblé dans des cellules de plantes, d'animaux et d'humains. Des variantes de la technique permettent également de réduire ou d'augmenter l'expression d'un gène choisi. Cette technologie peut donc être utilisée non seulement pour permettre de comprendre le rôle d'un gène, mais aussi de développer des thérapies pour des maladies héréditaires et acquises. <

Les technologies de modification du génome évoluent rapidement. II y a quelques années encore, les nucléases à doigts de zinc (en anglais zinc finger nucleases, ZFN) étaient la seule option à la disposition des chercheurs voulant modifier un génome. À la fin de 2011, une nouvelle méthode de modification des gènes, celle des TAL effector nucleases (TALEN) [45], a été découverte. Elle a été sélectionnée par la revue Nature Methods comme la «Méthode de l'année». Cette nouvelle technologie permettait de cibler des séquences de nucléotides plus facilement qu'avec des nucléases à doigts de zinc [46]. Dès janvier 2013, une autre méthode a cependant émergé à partir de travaux indépendants de plusieurs groupes de recherche. Cette nouvelle méthode (désignée sous le nom CRISPR, clustered regularly interspaced short palindromic repeats) utilisait une nucléase appelée Cas9 (CRISPR-associated protein 9) pour couper l'ADN et permettre l'édition du génome ${ }^{1}$ [47]. Les trois types de nucléases partagent le même mécanisme d'action: elles clivent I'ADN chromosomique de manière spécifique (Figure I) en déclenchant les systèmes endogènes de réparation de I'ADN introduisant une modification génomique ciblée.

De l'anglais genome editing, « réécriture du matériel génétique».

\section{CRISPR, \\ un système qui \\ permet de corriger \\ ou de modifier \\ l'expression de \\ gènes responsables \\ de maladies héréditaires}

\section{Jacques P. Tremblay}

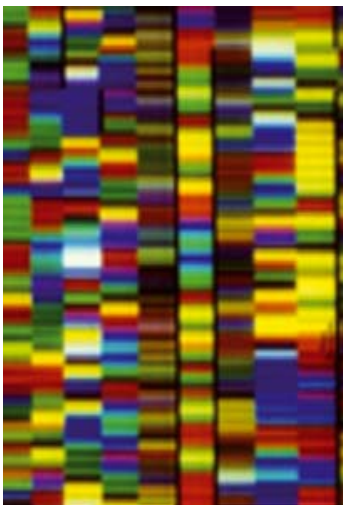

Département de Médecine

Moléculaire,

Faculté de Médecine,

Université Laval,

Centre de Recherche

du CHU de Québec,

2705 boulevard Laurier,

GIV Québec, Canada.

jacques-p.tremblay@crchul.

ulaval.ca

Chaque nucléase possède toutefois des caractéristiques uniques $[49,50](\rightarrow)$.

Les trois méthodes offrent la capacité aux chercheurs de modifier le génome des plantes, des animaux et même celui des cellules humaines [1]. La technologie CRISPR peut être utilisée en biotech- $(\rightarrow)$ Voir la Nouvelle de R. Gaudin et la Chronique génomique de B. Jordan, pages 959 et 1035 de ce numéro nologie, pour la recherche biomédicale et pour des applications thérapeutiques. Elle permet de modifier de façon spécifique la séquence des nucléotides d'un gène, ou d'un élément non codant (comme un microARN), pour les rendre non fonctionnel. Elle permet également d'éliminer ou d'introduire de un à plusieurs milliers de nucléotides afin de corriger une mutation responsable de maladie héréditaire. Les modifications apportées peuvent également conduire à identifier la fonction d'un gène qui est alors rendu non fonctionnel, et à évaluer les conséquences phénotypiques de mutations induites dans des cellules ou des organismes entiers [48].

\section{Survol du système CRISPR}

Le système CRISPR utilise un ARN guide ( $A R N g$ ) constitué d'une séquence constante de 42 nucléotides et d'une séquence variable de 20 nucléotides complémentaires d'une séquence choisie d'ADN (Figure 2A). Ce système est, à l'origine, présent chez les bactéries qui l'utilisent pour dégrader des séquences d'ADN non autologues 


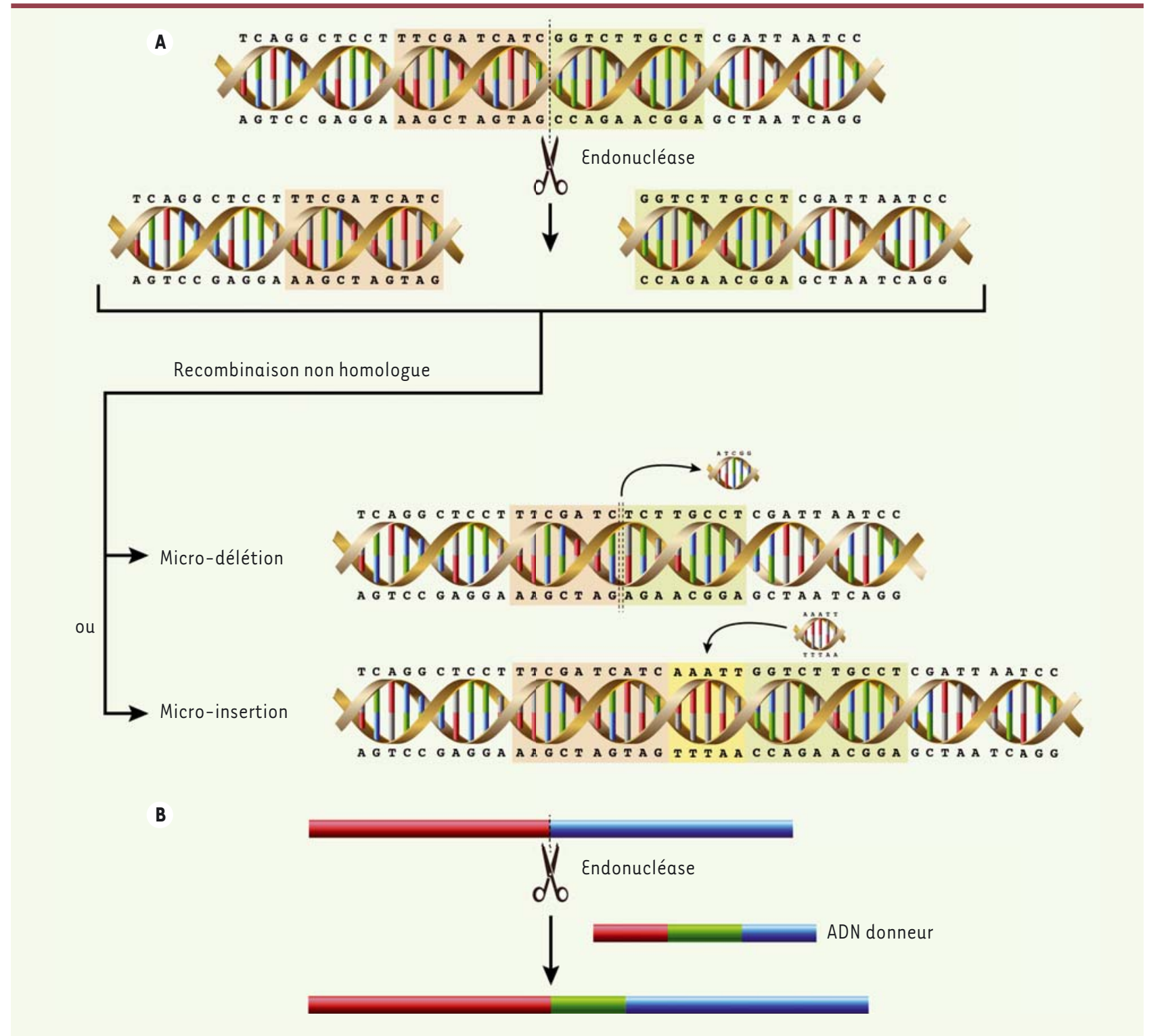

Figure 1. Principes de l'édition du génome à l'aide de nucléases. Plusieurs sortes d'endonucléases (méganucléases, nucléases à doigts de zinc, nucléases TAL effecteur et la combinaison d'un ARN guide et d'une Cas9) peuvent être conçues et produites par des méthodes de biologie moléculaire pour cibler une séquence choisie d'ADN afin d'y induire des coupures touchant les deux brins. Ces coupures peuvent être réparées soit par recombinaison non-homologue (A), soit par recombinaison homologue (B). Pour la recombinaison homologue, la présence d'une séquence d'ADN donneur est nécessaire. Cet ADN donneur doit contenir des séquences de nucléotides homologues (en rouge et bleu) à celles qui précèdent et suivent le site de coupure. Dans le schéma ces séquences sont courtes mais elles peuvent contenir jusqu'à 500 nucléotides. Entre les deux séquences homologues, l'ADN donneur peut contenir une séquence de nucléotides (en vert) plus ou moins longue qui permet de modifier quelques nucléotides du gène ciblé, d'y introduire un ou plusieurs exons manquants, ou même d'introduire un gène complet. La recombinaison non-homologue induit une réparation moins précise qui peut résulter soit en une micro-délétion, soit en une micro-insertion (en jaune) d’un nombre variable de nucléotides. Ces micro-insertions ou micro-délétions, si elles ne sont pas des multiples de trois nucléotides, changent le cadre de lecture du gène résultant soit en un gène non fonctionnel soit dans le rétablissement d'un gène fonctionnel lorsque le gène ciblé ne l'était pas.

provenant de plasmides ou de virus. C'est donc une sorte de système «immunitaire» bactérien qui leur permet de se protéger contre les infections virales [2]. À cette fin, les bactéries et les archées capturent de petits fragments d'ADN d'environ 30 paires de bases (appelés protospacers) provenant des phages ou des plasmides envahisseurs, et les insèrent dans leur propre génome. Ces séquences sont transcrites en ARN pré-CRISPR (pre-crRNA) et traitées pour donner naissance à un crARN (CRISPR-ARN). Il existe trois types de système CRISPR qui ciblent l'ARN ou l'ADN exogènes. Nous n'aborderons dans cette revue 

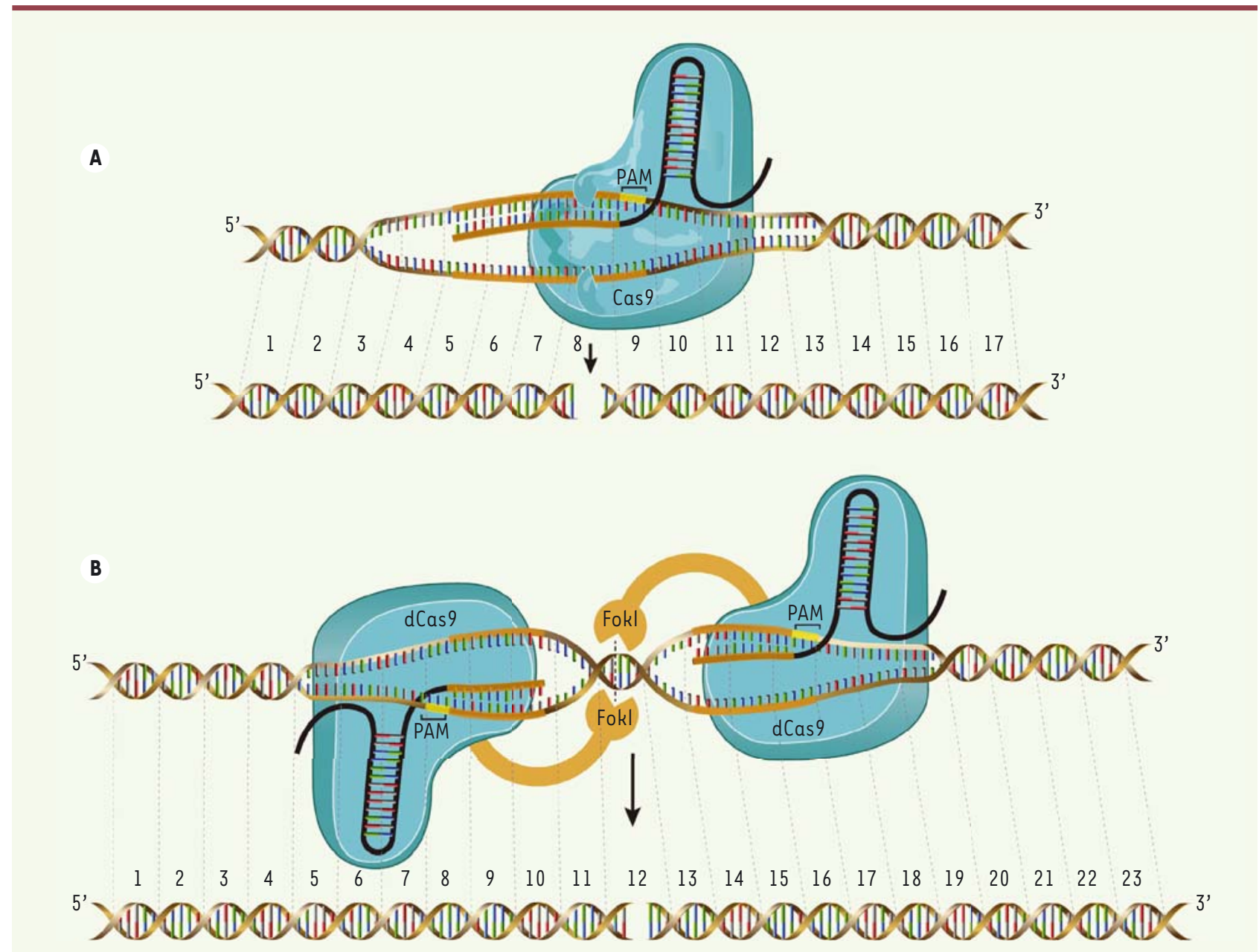

Figure 2. Représentation du système CRISPR/Cas9. A. Pour couper un gène avec la méthode CRISPR, il faut un ARN guide (ARNg) (noir et marron) complémentaire à une séquence de 17 à 20 nucléotides du gène ciblé. La seule restriction est que cette séquence doit être suivie par une séquence NGG ou NGA (appelée un PAM). L'ARNg forme un complexe avec l'ADN et la nucléase Cas9 (en bleu). La coupure par la nucléase se fait exactement trois nucléotides avant le PAM. Les lignes pointillées relient les séquences de nucléotides qui sont les mêmes avant et après la coupure. $B$. Pour éviter de couper des séquences d'ADN similaires à celle qui est ciblée, il est possible d'utiliser une nucléase Cas9 non-fonctionnelle (dCas9) qui est fusionnée avec la nucléase Fokl. La nucléase Fokl doit former un dimère pour couper l'ADN. Ce dimère ne peut être formé que si deux ARNg ciblent des séquences de nucléotides séparées de 13 à 17 nucléotides.

que le système de type II qui est le plus utilisé pour l'édition de gènes à des fins thérapeutiques.

Le système CRISPR de type II est composé de trois éléments : (1) le crARN qui est composé d'un ARN provenant de la transcription de I'ADN sauvegardé dans la matrice CRISPR et d'une partie d'une répétition ; (2) un second ARN présentant une complémentarité partielle avec la répétition, connu sous le nom de tracrARN (trans-activating crRNAs [tracrRNA]); et (3) la nucléase Cas9. Le complexe formé par le crARN et le tracrARN active la Cas9 et la guide vers sa cible. En 2012, Jinek et ses collègues [3] ont fusionné le crARN et le tracrARN de la bactérie Streptococcus pyogenes en un seul ARN guide (ARNg) qui induit de façon efficace le clivage par la Cas9 (Figure 2A). La Cas9 possède deux régions à activité nucléase, chacune permettant de cliver un brin de l'ADN ciblé. Elle se lie en premier à une séquence spécifique de 3 à 5 nucléotides appe- lée protospacer adjacent motif (PAM), située après la séquence ciblée par l'ARN guide, puis au complexe formé entre l'ARN guide et l'ADN. Pour la Cas9 provenant de $S$. pyogenes, le motif PAM est formé par les nucléotides NGG (nucléotide-guanine-guanine). Les PAM sont très fréquents dans tous les génomes. Ils ne limitent donc pas le nombre de séquences qui peuvent être ciblées. Il est aussi possible d'utiliser des Cas9 provenant d'autres bactéries qui possèdent des PAM différents.

Dès la publication de l'article de Jinek et ses collègues [3], les possibilités d'ingénierie du génome que présente le système CRISPR/Cas9 apparurent évidentes à plusieurs équipes. En janvier 2013, quatre groupes avaient déjà appliqué ce système à des cibles géno- 


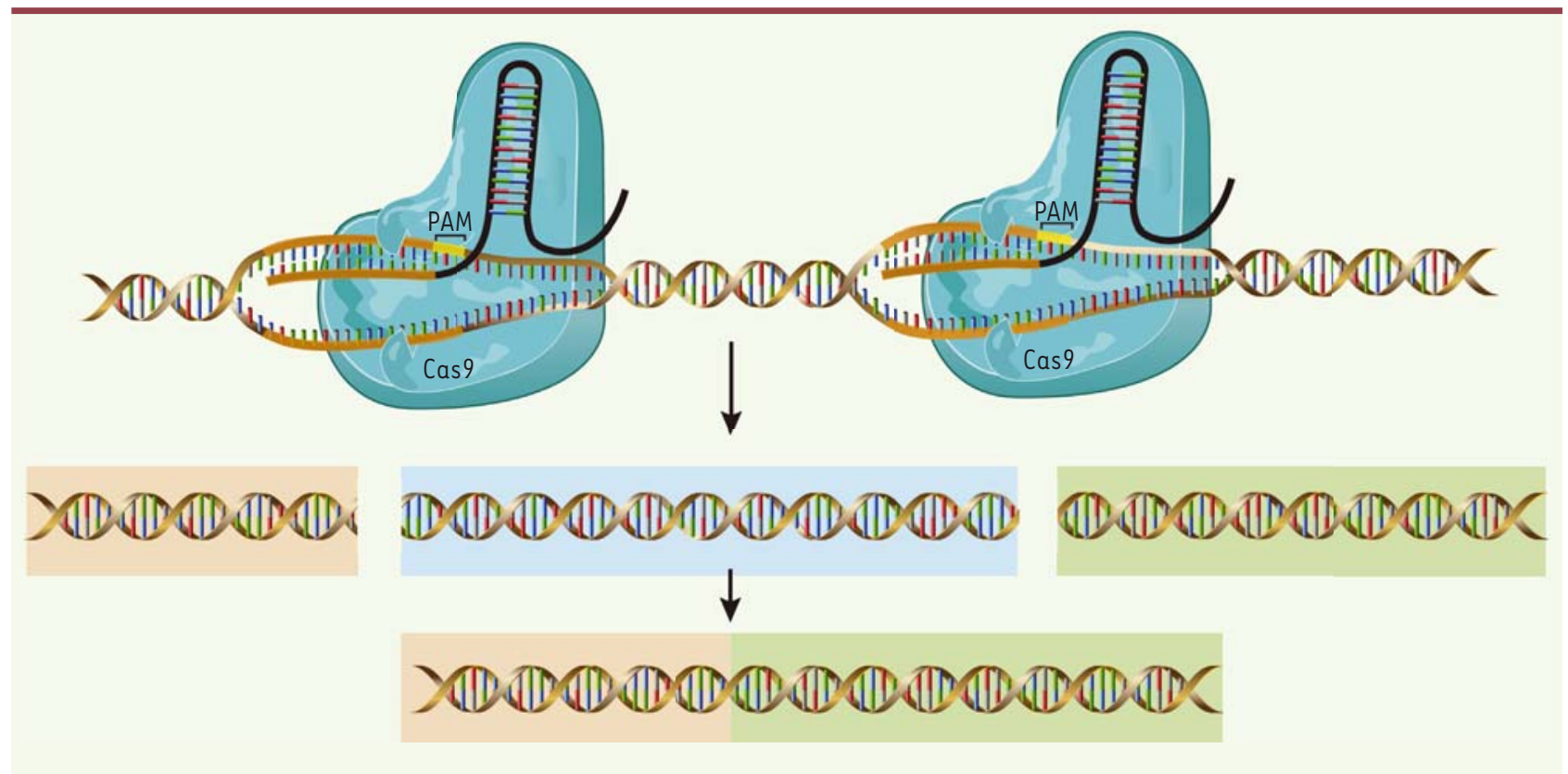

Figure 3. Utilisation du système CRISPR/Cas pour éliminer une séquence d'ADN. Il est possible de couper, en utilisant deux ARN guide (ARNg), les deux brins d'ADN à deux endroits dans un même chromosome. Ceci permet d'enlever une séquence de quelques nucléotides ou même de centaines de milliers de nucléotides.

miques présentes dans des cellules humaines [4-6] et Gratz et ses collaborateurs décrivaient son utilisation dans la drosophile [7]. D'autres publications ont relaté l'emploi du système pour induire des mutations ciblées dans les bactéries, les embryons de poisson zèbre et des cellules de mammifères. Depuis, des mutations ont été réalisées dans des plantes, des nématodes, des souris, des rats, des primates non humains et dans des cellules souches pluripotentes humaines.

Un consensus ressortant de ces différentes études est que l'ARN guide (ARNg) doit avoir une séquence de guidage de 17 à 20 nucléotides correspondant à I'ADN ciblé, suivie d'un motif constant correspondant au PAM spécifique de la Cas9 sélectionnée. L'ARNg est produit par transcription in vitro ou in vivo, et est habituellement associé aux promoteurs SP6, T3 et T7 (issus des bactériophages du même nom). L'ARN guide, ou l'ADN le codant, peut être introduit dans des cellules en utilisant, par exemple, des vecteurs viraux.

\section{Avantages du système CRISPR}

Les avantages du système CRISPR, comparé aux nucléases à doigts de zinc (ZFN) et aux TALEN, sont les suivants : (1) une seule protéine (Cas9) est nécessaire et, puisqu'elle est universelle, aucune ingénierie de protéines n'est nécessaire, contrairement aux méthodes utilisant les nucléases ZFN et les TALEN ; (2) le ciblage dépend de l'appariement des bases, de sorte que la conception des ARN guides nécessite uniquement une connaissance des règles de Watson et Crick ; (3) de nouveaux ARN guides sont très faciles à produire ; (4) il est possible de cibler différentes séquences simultanément en utilisant un mélange de plusieurs ARN guides; (5) contrairement aux nucléases ZFN et TALEN, le système CRISPR permet de cliver un ADN méthylé.

\section{Deux mécanismes de réparation de l'ADN}

Les cassures du double brin d'ADN produites par la Cas9 peuvent être réparées spontanément par jonction d'extrémités non-homologues (en anglais, non homologous end joining, NHEJ) ou en présence d'une séquence d'ADN donneur, par recombinaison homologue (en anglais, homology directed repair, HDR) (Figure I). Cet ADN donneur doit contenir, de chaque côté de la coupure de l'ADN ciblé, des séquences homologues allant de 50 à 2000 nucléotides. Entre ces deux séquences homologues, l'ADN donneur peut comporter une longue séquence de nucléotides à introduire dans le gène ciblé. Cette partie de I'ADN donneur permet ainsi d'induire une mutation d'un ou plusieurs nucléotides dans le gène qui a été ciblé, ou d'introduire des segments de gène qui en sont absents.

\section{Délivrance des composants du système CRISPR dans la cellule}

Pour les cellules en culture, les options pour l'introduction du gène qui code la protéine Cas 9 incluent la transfection d'ADN plasmidique, l'utilisation d'un vecteur viral et la transfection d'ARN messagers (ARNm) synthétiques. Le gène de la nucléase doit être encadré par un promoteur et une séquence de poly $A$ (succession de nucléotides adénosine). La protéine elle-même doit inclure un signal permettant son transport vers le noyau. Lorsque I'ARN guide et le gène de la protéine Cas9 sont délivrés à un 
organisme entier, d'autres méthodes, qui doivent être adaptées à chaque situation particulière, sont nécessaires. Dans certains cas, l'injection d'ARNm ou de l'ADN codant pour la nucléase est possible. C'est le cas chez les poissons zèbres, où les injections sont réalisées directement dans le cytoplasme de l'embryon peu après la fécondation. L'expression précoce des composants du système CRISPR conduit, après réparation par jonction des extrémités non homologues (NHE), non-homologous end joining), à des mutations somatiques et germinales. La même approche a été utilisée avec de nombreux autres organismes y compris des mammifères $[8,9]$. Pour tous les organismes, un ADN donneur peut être ajouté pour permettre une recombinaison homologue. L'efficacité de modification qui est ainsi obtenue dépend beaucoup du système utilisé et des séquences ciblées. En général, elle est bien inférieure au taux de mutations induites par NHEJ en l'absence de donneur.

\section{Les mutations induites par le système CRISPR}

Comme mentionné précédemment, lorsque la Cas9 clive avec succès le gène ciblé, des modifications spontanées sont introduites par jonction d'extrémités non-homologues. Ces modifications sont généralement des délétions et/ou des insertions d'une ou quelques dizaines de nucléotides. Les deux tiers d'entre elles vont induire un décalage du cadre de lecture dans une séquence codant la protéine et ainsi inactiver complètement le produit du gène. Si la modification se trouve dans une région critique de la protéine, un changement d'un multiple de 3 paires de bases peut également engendrer une protéine non fonctionnelle. Typiquement, la modification introduite dans la séquence est caractérisée par une amplification par PCR (polymerase chain reaction), suivie du séquençage de l'amplicon, ou de la digestion du produit de l'amplification par l'endonucléase $T 7 \varepsilon l^{2}$. Parmi les mutations induites par une coupure par la Cas9, la délétion d'un faible nombre de nucléotides est la plus fréquente. Ni la région hybride ARN/ADN, ni le PAM, ne sont épargnés par ces délétions. De temps en temps, des insertions et des suppressions de plusieurs centaines de paires de bases peuvent être produites.

Bien que chaque interruption soit habituellement réparée indépendamment, il est possible d'éliminer la séquence d'ADN présente entre deux coupures produites par deux ARN guides [10] (Figure 3). Dans le poisson zèbre, le porc, et les cellules humaines, des délétions importantes de plusieurs centaines de milliers de paires de bases, voire de sections de chromosomes, ont été obtenues par clivage au niveau de deux sites largement séparés [10,11].

\section{La recombinaison homologue (HDR) versus la réparation non homologue (NHEJ)}

Souvent, un expérimentateur souhaite modifier un gène par recombinaison homologue (HDR). Cela peut être difficile car, dans la plupart des situations, la réparation non homologue (NHEJ) prédomine dans la

${ }^{2}$ L'endonucléase $T 7 \varepsilon l$ permet de détecter des mutations car elle coupe les mésappariements des hétéroduplexes formés entre le brin sauvage et le brin muté après une étape de dénaturation/renaturation des produits de la PCR. correction des cassures d'ADN. Cette préférence varie selon les types de cellules. Lorsque les fréquences sont suffisamment élevées, les produits de recombinaison homologue souhaités peuvent être identifiés par des analyses moléculaires et un séquençage. Les recombinaisons homologues conduisent à des modifications très précises, ce qui est très utile pour corriger des gènes impliqués dans des maladies héréditaires. Elles peuvent également permettre d'introduire au niveau d'un gène une séquence codant pour un rapporteur afin de détecter la protéine qui en résulte.

\section{Coupures hors cible}

Plusieurs études initiales ont suggéré que le système CRISPR/Cas9 manquait de spécificité $[12,13]$. Ce problème a cependant été atténué récemment [14]. En principe, 20 paires de bases ( $p b$ ) sont plus que suffisantes pour être spécifiques d'un site cible unique dans le génome humain. Une hybridation parfaite de I'ARN et de I'ADN n'est cependant pas nécessaire pour qu'il y ait coupure par la Cas9. L'hybridation des nucléotides les plus proches du PAM est stricte alors que celle des nucléotides situés plus près du début de la séquence, en 5' de l'ARN, l'est moins et de multiples inadéquations sont apparemment tolérées. La protéine Cas9 stabilise probablement le duplex entre l'ARN guide et la cible. Elle détermine également les exigences de correspondance entre les nucléotides de l'ARN guide et ceux de l'ADN cible.

L'absence de nécessité d'un appariement parfait entre I'ARN guide et I'ADN cible dans le système CRISPR/Cas9 peut être un avantage adaptatif pour les bactéries lors de leur infection par un virus. Un appariement complet de 22 paires de bases n'est en fait pas nécessaire si le but est simplement d'obtenir une correspondance dans le génome viral de moins de $10^{6}$ paires de base, ce qui évite d'altérer des gènes de l'hôte. Les génomes viraux sont soumis à des variations constantes et à la sélection, de sorte que les virus produits différeront certainement légèrement de celui qui a mis en place un insert CRISPR. En permettant l'inadéquation entre l'ARN guide et l'ADN ciblé, la défense de l'hôte peut s'adapter à la variation du génome du virus.

Certains groupes $[13,15,16]$ ont décrit des modifications qui rendent le système CRISPR plus spécifique. Ils ont par exemple produit une version de la nucléase comportant un seul site actif, créant ainsi une Cas9 nickase (nCas9). Cette nCas9 ne coupe donc qu'un seul des deux brins d'ADN. Ils ont aussi utilisé deux ARN guides pour cibler simultanément une paire de séquences situées à proximité l'une de l'autre sur des brins opposés de la cible. Lorsque les deux sites sont coupés, une coupure double 


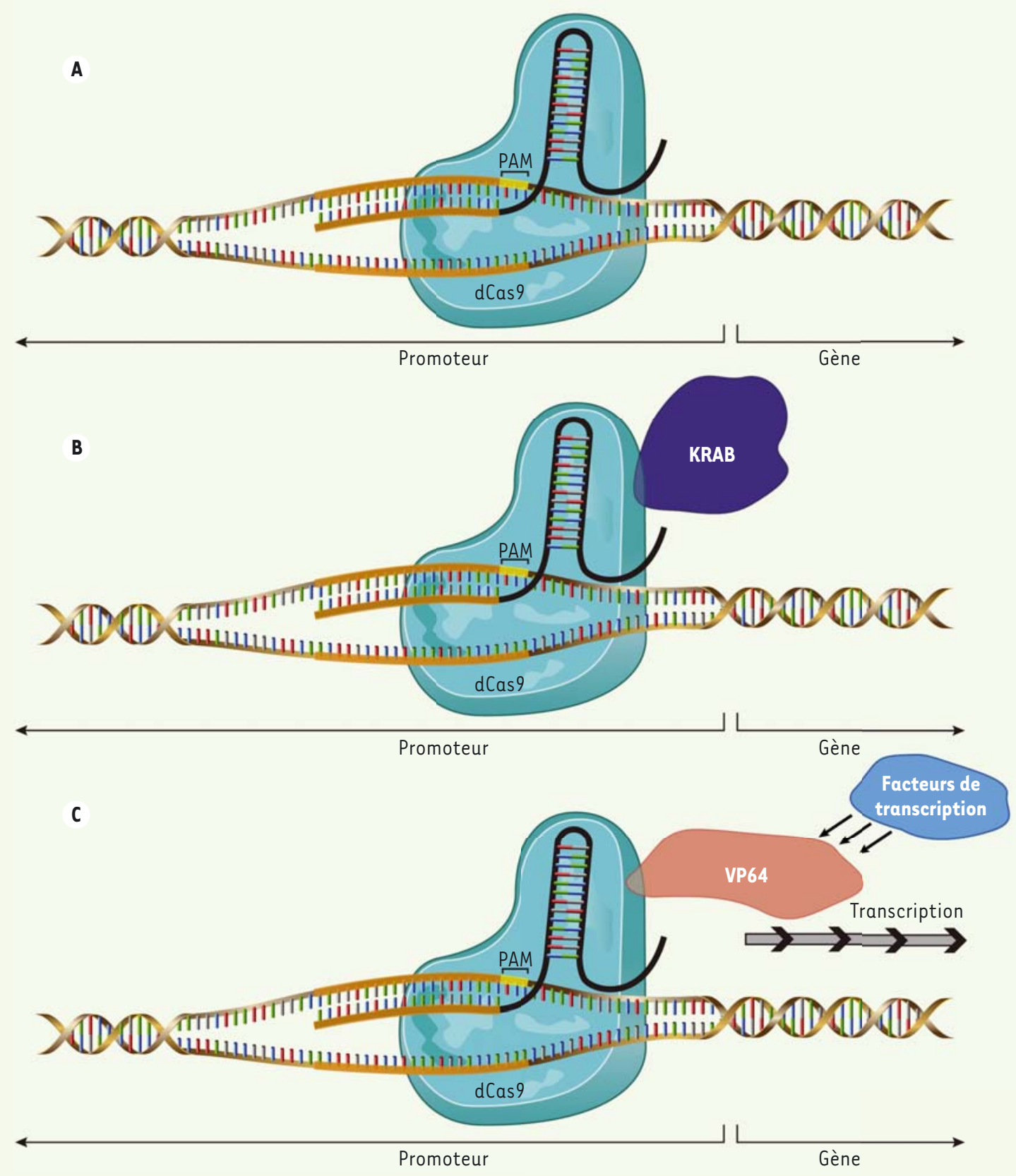

Figure 4. Modifications de l'expression d'un gène par le système CRISPR/Cas9. Le système CRISPR peut être utilisé pour réprimer ou induire l'expression d'un gène. Pour atteindre ce but, il faut cibler avec l'ARN guide (ARNg) une séquence de nucléotides dans le promoteur du gène à modifier et utiliser une protéine Cas9 non fonctionnelle (dCas9). A. La simple présence de la protéine dCas9 diminue l'expression du gène. B. II est aussi possible de fusionner la protéine dCas9 avec un inhibiteur de la transcription tel que la protéine KRAB. C. Pour augmenter l'expression d'un gène, la protéine dCas9 peut être fusionnée au peptide viral VP64 qui recrute ensuite des facteurs de transcription.

brin est ainsi produite permettant la réparation par jonction d'extrémités non-homologues (NHEJ) ou homologues (HDR). Cette approche qui nécessite deux coupures rapprochées augmente ainsi la spécificité de la méthode. Fu et al. [17] ont constaté que, dans plusieurs cas, des ARN guides légèrement tronqués (17 ou 18 nucléotides), en comparaison de la séquence standard de guidage de 20 nucléotides, présentent une meilleure spécificité. Finalement le groupe de J.K. Joung [18] a utilisé une nucléase Cas9 non fonctionnelle (dCas9) fusionnée avec la nucléase Fokl de Flavobacterium okeanokoites (Figure 2B). Cette nucléase a déjà été utilisée avec les protéines à doigts de zinc (ZFN) et les TALEN. Elle nécessite la formation d'un dimère pour couper les deux brins de I'ADN. Pour former un tel dimère il est nécessaire d'utiliser, avec la dCas9-Fokl, deux ARN guides ciblant 
des séquences séparées de 13 à 17 nucléotides. Cette modification du système CRISPR/Cas permet de cibler avec précision un gène et réduit grandement les mutations hors cible.

\section{Contrôle de l'expression des gènes}

Le système CRISPR/Cas9 peut être utilisé pour contrôler de façon spécifique l'expression d'un gène (Figure 4). Dans une première approche, Qi et ses collègues [19] ont utilisé la nucléase Cas9 mutée non fonctionnelle (dCas9) pour diminuer l'expression d'un gène ciblé. L'attachement de I'ARN guide et de la dCas9 à l'ADN bloque en effet l'élongation de l'ARN messager, ainsi que l'attachement de l'ARN polymérase et des facteurs de transcription. Par la suite, Gilbert et ses collègues [20] ont fusionné la protéine Cas9 avec des fragments de protéines qui modifient la chromatine, comme le domaine KRAB (Kruppel-associated suppression box) de la protéine à doigts de zinc humaine Koxl (ou zinc finger protein 10), afin de réprimer l'expression d'un gène dans les cellules de levure et dans des cellules humaines. Ce domaine KRAB provoque une inhibition spécifique des ARN polymérases II et III.

Le système CRISPR/Cas9 peut être utilisé pour augmenter l'expression d'un gène en fusionnant la protéine non fonctionnelle dCas9 avec un activateur de la transcription comme VP64 ${ }^{3}$. En utilisant la dCas9-VP64 avec un ARN guide ciblant le promoteur d'un gène, Maeder et ses collaborateurs ont ainsi réussi à augmenter l'expression de plusieurs gènes humains [21]. Les groupes de Gersbach [22], Jaenisch [23] et Joung [21] ont également montré que l'utilisation de la dCas9-VP64 avec plusieurs ARN guides ciblant le même promoteur permettait d'obtenir un effet synergique sur l'expression d'un gène humain. Nous avons utilisé cette approche afin d'augmenter l'expression de la frataxine, une petite protéine de la matrice mitochondriale dont la déficience est responsable, chez l'homme, de l'ataxie de Friedreich ${ }^{4}$ (résultats non publiés). Hilton et collaborateurs ont aussi montré qu'il est possible d'augmenter encore plus l'expression d'un gène en fusionnant la dCas9 avec une acétyltransférase afin de modifier les histones [24]. L'induction de l'expression d'un gène spécifique impliqué dans une pathologie pourrait ainsi avoir une application thérapeutique.

\section{Applications à des organismes modèles}

Nous ne pouvons décrire ici tous les organismes et types de cellules dans lesquels le système CRISPR/Cas9 a déjà été appliqué avec succès. Nous allons seulement en présenter quelques exemples.

L'un est I'application du système CRISPR/Cas9 au poisson zèbre. Malgré la popularité de ce modèle animal pour les études génétiques et biochimiques, aucune procédure efficace de manipulation de gènes n'était en effet disponible avant l'avènement du système CRISPR/Cas9. Ce système a permis de muter simultanément plusieurs gènes chez ces poissons $[7,25]$. II a également été possible de muter des gènes par

\footnotetext{
${ }^{3}$ VP64 est un activateur transcriptionnel composé de 4 copies de la protéine VP16 du virus de l'herpes (herpes simplex viral protein 16).

${ }^{4}$ L'ataxie de Friedreich est une maladie neurodégénérative héréditaire qui se manifeste par une perte
} progressive de la motricité. recombinaisons homologues mais avec une efficacité très faible [26].

Chez la souris, Wang et ses collaborateurs [27] ont montré l'exceptionnelle puissance du système en exprimant la protéine Cas9 et jusque cinq ARN guides différents dans des cellules souches embryonnaires. Cette expérience a conduit à l'obtention de cellules possédant des perturbations simultanées dans tous les allèles des gènes ciblés. Chez les embryons de souris, la co-injection de l'ARN messager de la Cas9 avec deux ARN guide a permis la mutation bi-allélique de deux gènes avec une grande efficacité. La co-injection d'un ADN donneur a permis, de plus, d'obtenir des changements de séquence au sein des deux gènes. D'autres applications se sont intéressées à des modèles de pathologies. Wu et ses collaborateurs [28] ont ainsi utilisé la technologie CRISPR/Cas9 pour corriger le gène Crygc (gamma C-crystallin gene) dont l'altération est à l'origine de cataracte héréditaire chez la souris. D'autres groupes ont muté des gènes afin de produire des modèles de cancers $[29,30]$. De même, le gène codant la dystrophine a été muté pour créer un modèle de la dystrophie de Duchenne chez le rat [31]. II a également a été corrigé dans un modèle murin de cette même pathologie [32]. Enfin, il a été possible de muter le génome de Plasmodium Yolii, le parasite responsable de la malaria dans un modèle murin [33].

Des modèles de maladies héréditaires humaines ont également été créés chez le porc [34] et le singe [35] grâce à la technologie CRISPR/Cas9.

\section{Applications humaines}

La recherche portant sur l'ingénierie des génomes reposant sur les nucléases a mis l'accent sur l'utilisation de cellules humaines en culture, en raison des applications potentielles en santé humaine. Le système CRISPR/ Cas9 permet de réaliser un ciblage simple de gènes et d'établir facilement des lignées cellulaires modifiées stables. Deux groupes ont récemment montré la capacité de multiplexage du système CRISPR/Cas en ciblant simultanément des dizaines de milliers de gènes dans des cultures de cellules humaines [27, 36]. Schwank et ses collaborateurs [37] ont, quant à eux, utilisé le système CRISPR/Cas9 pour corriger, dans des cellules souches pluripotentes humaines, le gène CFTR (cystic fibrosis transmembrane conductance regulator) dont la mutation est à l'origine de la mucoviscidose.

\section{Regard vers l'avenir}

Le système CRISPR/Cas9 est un outil précieux pour la manipulation génétique. À ce jour, plusieurs études ont 
mis l'accent sur l'optimisation de la construction de ces nucléases et la démonstration de leur activité. Les applications pratiques sont maintenant en développement dans les domaines de la médecine et la production d'aliments. Grâce à des mutations spécifiques réalisées dans le génome, on peut s'attendre, désormais, à voir apparaître de plus en plus d'études concernant la fonction de gènes et le développement de modèles de pathologies humaines. Des articles récents ont déjà montré qu'il était possible d'utiliser le système CRISPR/Cas9 pour détruire un provirus intégré dans le génome d'un patient [38, 39].

Afin d'élargir les applications possibles du système CRISPR/Cas9, I'utilisation de nucléases Cas 9 provenant d'autres organismes que $S$. pyogenes peut s'avérer utile $[40,41]$. Les nouveaux motifs PAM correspondants pourraient ainsi être plus souples ou plus stricts, ce qui permettrait d'élargir la gamme de sites accessibles. Certaines protéines Cas9 pourraient également être moins tolérantes à l'inadéquation entre l'ADN cible et l'ARN guide. La découverte d'une Cas9 plus petite a déjà permis de délivrer, in vivo dans des souris, toutes les composantes du système CRISPR/Cas9 avec un seul vecteur viral (virus adéno-associé) et de modifier ainsi un gène impliqué dans le métabolisme du cholestérol [42]. Ce travail a ouvert la voie des applications de correction in vivo pour de nombreux gènes responsables de maladies héréditaires. Cependant, la modification récente d'embryons humains avec le système CRISPR/Cas9 a montré que l'utilisation de cette technologie peut aussi soulever des controverses [43, 48]. Un groupe de chercheurs a d'ailleurs plaidé en faveur d'une utilisation prudente de cette technologie chez l'homme [44]. $\diamond$

\section{SUMMARY}

The CRISPR system can correct or modify the expression of genes responsible for hereditary diseases

A new technology, called CRISPR, derived from the immune system of bacteria, uses a Cas9 nuclease and a guided RNA complementary to a 20 nucleotides sequence of a gene to induce double strand DNA breaks. This permits to modify specifically the targeted gene in plant, animal and human cells. Variants of the technique also permit to reduce or increase the expression of a selected gene. This technology may thus be used not only to understand the role of a gene but also to develop therapies for hereditary and acquired diseases. $\diamond$

\section{LIENS D'INTÉRÊT}

L'auteur déclare n'avoir aucun lien d'intérêt concernant les données publiées dans cet article.

\section{RÉFÉRENCES}

1. Jinek M, Jiang F, Taylor DW, et al. Structures of Cas9 endonucleases reveal RNA-mediated conformational activation. Science $2014 ; 343: 1247997$.

2. Fineran PC, Charpentier $\varepsilon$. Memory of viral infections by CRISPR-Cas adaptive immune systems: acquisition of new information. Virology $2012 ; 434$ : 202-9.

3. Jinek M, Chylinski K, Fonfara I, et al. A programmable dual-RNA-guided DNA endonuclease in adaptive bacterial immunity. Science 2012 ; $337: 816-21$.

4. Mali P, Yang L, Esvelt KM, et al. RNA-guided human genome engineering via Cas9. Science 2013 ; $339: 823-6$.

5. Cong L, Ran FA, Cox D, et al. Multiplex genome engineering using CRISPR/Cas systems. Science $2013 ; 339: 819-23$.
6. Cho SW, Kim S, Kim JM, Kim JS. Targeted genome engineering in human cells with the Cas9 RNA-guided endonuclease. Nat Biotechnol $2013 ; 31: 230-2$.

7. Gratz SJ, Cummings AM, Nguyen JN, et al. Genome engineering of Drosophila with the CRISPR RNA-guided Cas9 nuclease. Genetics 2013 ; 194 : 1029-35.

8. Fu Y, Foden JA, Khayter C, et al. High-frequency off-target mutagenesis induced by CRISPR-Cas nucleases in human cells. Nat Biotechnol $2013 ; 31$ 822-6.

9. Cradick TJ, Fine EJ, Antico CJ, Bao G. CRISPR/Cas9 systems targeting betaglobin and CCR5 genes have substantial off-target activity. Nucleic Acids Res $2013 ; 41:$ 9584-92.

10. Zheng $Q$, Cai X, Tan MH, et al. Precise gene deletion and replacement using the CRISPR/Cas9 system in human cells. Biotechniques $2014 ; 57: 115-24$.

11. He Z, Proudfoot C, Mileham AJ, et al. Highly efficient targeted chromosome deletions using CRISPR/Cas9. Biotechnol Bioeng $2015 ; 112$ : 1060-4.

12. Li D, Qiu Z, Shao Y, et al. Heritable gene targeting in the mouse and rat using a CRISPR-Cas system. Nat Biotechnol 2013 ; $31: 681-3$.

13. Sung YH, Kim JM, Kim HT, et al. Highly efficient gene knockout in mice and zebrafish with RNA-guided endonucleases. Genome Res 2014 ; 24 : 125-31.

14. Tan EP, Li Y, Velasco-Herrera Mdel C, et al. Off-target assessment of CRISPRCas9 guiding RNAs in human iPS and mouse ES cells. Genesis 2015 ; 53 : 225-36.

15. Li W, Teng F, Li T, Zhou Q. Simultaneous generation and germline transmission of multiple gene mutations in rat using CRISPR-Cas systems. Nat Biotechnol $2013 ; 31: 684-6$.

16. Ran FA, Hsu PD, Lin CY, et al. Double nicking by RNA-guided CRISPR Cas 9 for enhanced genome editing specificity. Cell 2013 ; 154 : 1380-9.

17. Fu Y, Sander JD, Reyon D, et al. Improving CRISPR-Cas nuclease specificity using truncated guide RNAs. Nat Biotechnol $2014 ; 32$ : 279-84.

18. Tsai SQ, Wyvekens N, Khayter C, et al. Dimeric CRISPR RNA-guided Fokl nucleases for highly specific genome editing. Nat Biotechnol $2014 ; 32$ : 569-76.

19. Di LS, Larson MH, Gilbert LA, et al. Repurposing CRISPR as an RNA-guided platform for sequence-specific control of gene expression. Cell $2013 ; 152$ : 1173-83.

20. Gilbert LA, Larson MH, Morsut L, et al. CRISPR-mediated modular RNAguided regulation of transcription in eukaryotes. Cell $2013 ; 154: 442-51$.

21. Maeder ML, Linder SJ, Cascio VM, et al. CRISPR RNA-guided activation of endogenous human genes. Nat Methods $2013 ; 10: 977-9$.

22. Perez-Pinera $P$, Kocak DD, Vockley CM, et al. RNA-guided gene activation by CRISPR-Cas9-based transcription factors. Nat Methods 2013 ; 10 : 973-6.

23. Cheng AW, Wang $\mathrm{H}$, Yang $\mathrm{H}$, et al. Multiplexed activation of endogenous genes by CRISPR-on, an RNA-guided transcriptional activator system. Cell Res $2013 ; 23$ : 1163-71.

24. Hilton IB, D'Ippolito AM, Vockley CM, et al. Epigenome editing by a CRISPRCas9-based acetyltransferase activates genes from promoters and enhancers. Nat Biotechnol $2015 ; 33: 510-7$.

25. Hwang WY, Fu Y, Reyon D, et al. Efficient genome editing in zebrafish using a CRISPR-Cas system. Nat Biotechnol 2013 ; $31: 227-9$.

26. Irion U, Krauss J, Nusslein-Volhard C. Precise and efficient genome editing in zebrafish using the CRISPR/Cas9 system. Development 2014 ; 141 : 4827-30.

27. Wang T, Wei JJ, Sabatini DM, Lander ES. Genetic screens in human cells using the CRISPR-Cas9 system. Science $2014 ; 343: 80-4$.

28. Wu $Y$, Liang $D$, Wang $Y$, et al. Correction of a genetic disease in mouse via use of CRISPR-Cas9. Cell Stem Cell 2013 ; $13:$ 659-62.

29. Heckl D, Kowalczyk MS, Yudovich D, et al. Generation of mouse models of myeloid malignancy with combinatorial genetic lesions using CRISPR-Cas9 genome editing. Nat Biotechnol $2014 ; 32$ : 941-6.

30. Xue W, Chen S, Yin H, et al. CRISPR-mediated direct mutation of cancer genes in the mouse liver. Nature $2014 ; 514: 380-4$.

31. Nakamura K, Fujii W, Tsuboi M, et al. Generation of muscular dystrophy model rats with a CRISPR/Cas system. Sci Rep 2014; $4: 5635$.

32. Long C, McAnally JR, Shelton JM, et al. Prevention of muscular dystrophy in mice by CRISPR/Cas9-mediated editing of germline DNA. Science 2014; $345: 1184-8$.

33. Zhang $C$, Xiao $B$, Jiang $Y$, et al. Efficient editing of malaria parasite genome using the CRISPR/Cas9 system. MBio 2014 ; 5 : e01414-14.

34. Whitworth KM, Lee K, Benne JA, et al. Use of the CRISPR/Cas9 system to produce genetically engineered pigs from in vitro-derived oocytes and embryos. Biol Reprod $2014 ; 91: 78$.

35. Niu Y, Shen B, Cui Y, et al. Generation of gene-modified cynomolgus monkey via Cas9/RNA-mediated gene targeting in one-cell embryos. Cell $2014 ; 156$ : 836-43. 


\section{RÉFÉRENCES}

36. Shalem 0 , Sanjana NE, Hartenian $\varepsilon$, et al. Genome-scale CRISPR-Cas9 knockout screening in human cells. Science $2014 ; 343: 84-7$.

37. Schwank G, Koo BK, Sasselli V, et al. Functional repair of CFTR by CRISPR/Cas9 in intestinal stem cell organoids of cystic fibrosis patients. Cell Stem Cell $2013 ; 13: 653-8$.

38. Ebina H, Misawa N, Kanemura Y, Koyanagi Y. Harnessing the CRISPR/Cas9 system to disrupt latent HIV-1 provirus. Sci Rep $2013 ; 3: 2510$.

39. Hu W, Kaminski R, Yang F, et al. RNA-directed gene editing specifically eradicates latent and prevents new HIV-1 infection. Proc Natl Acad Sci USA 2014 ; 111 : 11461-6.

40. Hou Z, Zhang $\mathrm{Y}$, Propson NE, et al. Efficient genome engineering in human pluripotent stem cells using Cas9 from Neisseria meningitidis. Proc Natl Acad Sci USA 2013 ; 110 : 15644-9.

41. Fonfara I, Le Rhun A, Chylinski K, et al. Phylogeny of Cas9 determines functional exchangeability of dual-RNA and Cas9 among orthologous type II CRISPR-Cas systems. Nucleic Acids Res 2014 ; $42: 2577-90$

42. Ran FA, Cong L, Yan WX, et al. In vivo genome editing using Staphylococcus aureus Cas9. Nature $2015 ; 520: 186-91$

43. Liang $\mathrm{P}, \mathrm{Xu} Y$, Zhang $\mathrm{X}$, et al. CRISPR/Cas9-mediated gene editing in human tripronuclear zygotes. Protein Cell $2015 ; 6: 363-72$.

44. Baltimore $D$, Berg $P$, Botchan $M$, et al. Biotechnology. A prudent path forward for genomic engineering and germline gene modification. Science $2015 ; 348: 36-8$.
45. Dion S, Demattei MV, Renault S. Les domaines à doigts de zinc. Med Sci (Paris) $2007 ; 23: 834-9$

46. Dupret $\mathrm{B}$, Angrand PO. L'ingénierie des génomes par les TALEN. Med Sci (Paris) $2014 ; 30: 186-93$.

47. Gilgenkrantz H. La révolution des CRISPR est en marche. Med Sci (Paris) $2014 ; 30: 1066-9$.

48. Jordan B. Thérapie génique germinale, le retour? Med Sci (Paris) 2015 ; 31 691-5.

49. Gaudin R. Améliorons notre expérience de la molécule unique grâce à CRISPR. Med Sci (Paris) $2015 ; 31: 959-61$.

50. Jordan B. CRISPR-Cas9, une nouvelle donne pour la thérapie génique. Med Sci (Paris) $2015 ; 31: 1035-8$

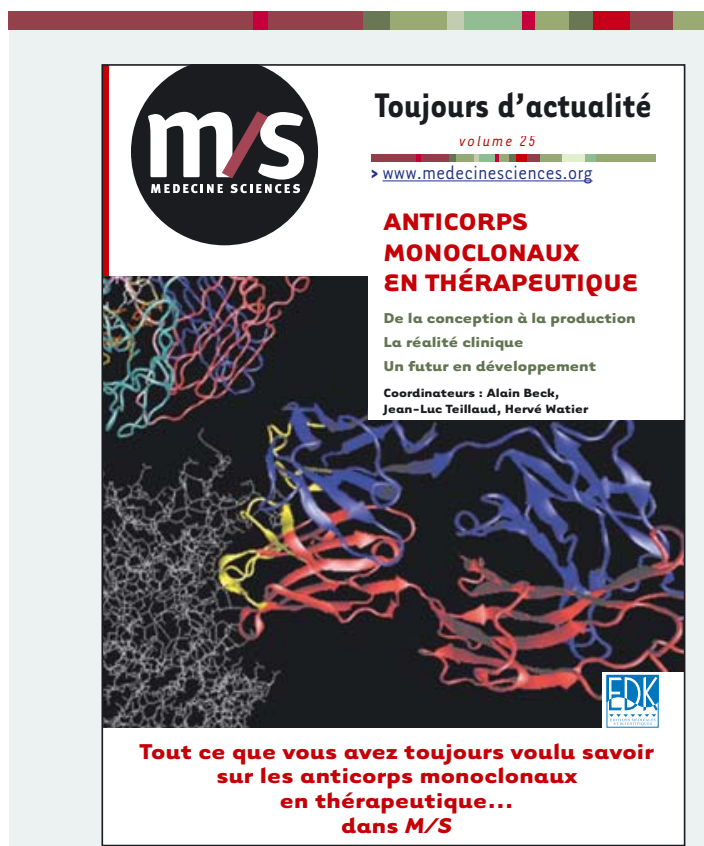

Tout ce que vous avez toujours voulu savoir sur les anticorps monoclonaux en thérapeutique... dans Médecine/Sciences. Pourquoi un numéro spécial de Médecine/Sciences sur les anticorps monoclonaux thérapeutiques? II nous a semblé que le moment était venu de dresser un état des lieux de ces biomédicaments qui prennent désormais une place considérable - et croissante dans les traitements de maladies souvent lourdes et désespérantes. Ce voyage que nous vous proposons à la découverte du monde des anticorps thérapeutiques nous a appris, ou plutôt rappelé, une évidence : les compétences en France sont fortes et nombreuses, qu'elles soient académiques ou industrielles, biotechnologiques ou cliniques. Le paysage français, trop longtemps discret, bruisse désormais de mille initiatives balayant de multiples aspects des anticorps thérapeutiques : études précliniques et cliniques menées avec de nouveaux anticorps dirigés contre des cibles originales, développement de nouveaux formats d'anticorps ou d'anticorps optimisés reposant sur des études structurales et fonctionnelles sophistiquées, recherche active de cibles pertinentes, mise au point de méthodologies de bioproduction, de couplage, etc. L'expansion industrielle rapide de ce champ est un défi que peut et doit relever notre pays, défi tant scientifique qu'économique, avec ses combats pour la propriété intellectuelle et pour l'emploi de nos jeunes scientifiques.

Alain Beck, Jean-Luc Teillaud, Hervé Watier

\section{À retourner à EDK, 109, avenue Aristide Briand, 92541 Montrouge Cedex, France}

Tél. : 0141177405 - Fax : 0149850345 - E-mail : edk@edk.fr

NOM :

Prénom

Adresse :

Code postal :

Ville :

Pays :

Fonction :

Je souhaite recevoir $\mathbf{M} / \mathbf{S} \mathbf{n}^{\circ} \mathbf{1 2}$ - décembre 2009 (Anticorps monoclonaux en thérapeutique) : $25 €+3 €$ de port $=\mathbf{2 8} € \mathbf{T T C}$ en exemplaire, soit un total de $€$

Par chèque, à l'ordre de $\mathbf{E} \mathbf{D} \mathbf{K}$

Par carte bancaire :

Visa

$\square$ Eurocard/Mastercard

Carte $n^{\circ}$

Date d'expiration :

$\mathrm{N}^{\circ}$ de contrôle au dos de la carte :

Signature : 